\title{
Anchoring glycine receptors
}

\section{Stanley C. Froehner}

WORKING out how synapses are formed has occupied neurobiologists for many years. On page 745 of this issue ${ }^{1}$ Kirsch $e t$ al. now report a major step in understanding how membrane proteins are reorganized during this process. They show that a peripheral membrane protein, termed 'gephyrin' (from the Greek for 'bridge'), is essential for glycine receptor clustering at inhibitory synapses.

Consider the complexity of synapse formation in the brain. A single neuron often receives synaptic input from thousands of presynaptic axons. And exquisite coordination between the pre- and postsynaptic cells is required to achieve proper alignment of the presynaptic terminals with postsynaptic sites, which possess high densities of the correct neurotransmitter receptor, thus ensuring rapid and specific synaptic transmission. The neuromuscular junction, where extremely high concentrations of nicotinic acetylcholine receptors are confined to the muscle synaptic membrane, has attracted particular attention in the expectation that principles established at this synapse will be applicable to the central nervous system. Kirsch et al., in contrast, took the direct approach by studying the glycinergic synapse in the spinal cord.

Glycine receptors mediate synaptic inhibition in the spinal cord by gating chloride ions ${ }^{2}$. Like other members of the ligand-gated ion channel family, the glycine receptor is a pentameric oligomer composed of two subunits, the ligandbinding $\alpha$-subunit and a structural $\beta$ subunit. Both subunits span the membrane several times and together make up the core of the functional complex. A third protein, gephyrin (formerly known as the $93 \mathrm{~K}$ protein) is closely associated with the glycine receptor core and copurifies with it. Gephyrin is a peripheral membrane protein associated with the cytoplasmic side of the postsynaptic membrane. This location, and the tendency of gephyrin to form large aggregates, prompted the suggestion that it might serve as an anchor and immobilize glycine receptors at the synapse.

As a direct test of this idea, Kirsch et al. used antisense oligonucleotides to deplete neurons of gephyrin. Rat spinalcord neurons in culture express glycine receptors, which are initially localized intracellularly. After a few days, the receptors appear on the cell surface, and become organized into small clusters (thought to be sites of synaptic contact) on the cell body and along the neurites. Gephyrin aggregates appear before the receptor clusters, but eventually the two become colocalized.

Kirsch et al. treated neuronal cultures with an antisense oligonucleotide spanning the start codon of the gephyrin messenger RNA and found that expression of gephyrin protein was decreased by more than 95 per cent. Concomitantly, the

derived in part from the innervating neuron, direct clustering of nicotinic acetylcholine receptors in the postsynaptic membrane. Gephyrin is not present at this synapse, but another peripheral membrane protein, the $43 \mathrm{~K}$ protein, is closely associated with cytoplasmic domains of the acetylcholine receptor and may subserve a similar role (for review see ref. 4). Recombinant $43 \mathrm{~K}$ protein forms membrane-associated clusters when expressed in non-muscle cells, and acetylcholine receptors become associated with $43 \mathrm{~K}$ protein clusters when the two proteins are coexpressed. Association between the $43 \mathrm{~K}$ protein and actin has been proposed as a mechanism for immobilizing acetylcholine receptors at the neuromuscular synapse. So although the proteins involved are different (gephyrin and the $43 \mathrm{~K}$ protein share no similarities in amino-acid sequence), the general concept that a peripheral membrane protein links neurotransmitter receptors to the submembrane cytoskeleton may apply at both synapses.

Gephyrin could be involved with other neurotransmitter receptors. It is expressed widely in the nervous system, even in some regions of the brain which lack glycine receptor $\alpha$ subunits ${ }^{5}$. In this respect, the existence of several forms of gephyrin generated by alternative splicing of RNA transcripts may provide some hints ${ }^{6}$. Selective use of four different cassettes, encoding sequences of $14,16,26$ and 36 amino

number of cells with glycine receptor clusters, and the number of clusters in each cell, were dramatically reduced. The effect was reversible and specific (there were no alterations in cell morphology or in expression of glycine receptors, and sense oligonucleotides had no effect). Gephyrin, then, is essential for the formation of glycine receptor clusters.

How it does this is not known, but gephyrin's ability to interact with the cytoskeleton is likely to be important. Several lines of evidence show that it binds polymerized tubulin ${ }^{3}$. The interaction between gephyrin and tubulin is a high-affinity, cooperative one, which approaches a stoichiometry of $1: 4$ at saturation. So gephyrin may provide a link between glycine receptors and the subsynaptic tubulin, thereby immobilizing receptors into clusters within the postsynaptic membrane.

How general is such a mechanism likely to be? Synaptic receptor clustering has been most thoroughly studied at the neuromuscular junction, where basal lamina proteins such as agrin, which are acids, could produce a large number of functionally diverse proteins. The cassettes are restricted to the amino-terminal half of the protein, raising the possibility that this variable part associates with different membrane receptors, depending on the cassettes used, while the constant carboxy-terminal region of the protein binds microtubules. As the biochemical characterization of other ligand-gated synaptic receptors advances, it will be interesting to learn if they associate with particular isoforms of gephyrin, or whether each receptor type has its own unique peripheral membrane protein.

Stanley C. Froehner is in the Department of Physiology, University of North Carolina, Chapel Hill, North Carolina 27599, USA.

\footnotetext{
Kirsch, J., Wolters, I., Triller, A. \& Betz, H. Nature 366 745-748 (1993).

Langosch, D., Becker, C.-M. \& Betz, H. Eur. J. Biochem 194, 1-8(1990).

3. Kirsch, J. et al. J. biol. Chem. 266, 22242-22245 (1991).

4. Froehner, S. C. A. Rev. Neurosci. 16, 347-368 (1993)

5. Kirsch. J., Malosio, M.-L., Wolters, I. \& Betz, H. Eur. J. Neurosci. 5, 1109-1117 (1993).

6. Prior, P.etal. Neuron 8, 1161-1170 (1992),
} 